OPEN ACCESS

Edited by:

Sanjeev Kumar Srivastava, Mitchell Cancer Institute,

United States

Reviewed by:

Megha Verma,

Janssen Pharmaceuticals, Inc.

United States

Vishwa Khare,

Eurofins Viracor, United States

*Correspondence:

Tao Fu

tfu001@whu.edu.cn

${ }^{\dagger}$ These authors have contributed equally to this work

Specialty section This article was submitted to

RNA,

a section of the journal

Frontiers in Genetics

Received: 21 January 2020

Accepted: 24 April 2020

Published: 03 June 2020

Citation:

Song W, Ren J, Wang C, Ge Y and Fu T (2020) Analysis of Circular RNA-Related Competing Endogenous RNA Identifies the Immune-Related Risk Signature for Colorectal Cancer.

Front. Genet. 11:505.

doi: 10.3389/fgene.2020.00505

\section{Analysis of Circular RNA-Related Competing Endogenous RNA Identifies the Immune-Related Risk Signature for Colorectal Cancer}

\author{
Wei Songt, Jun Rent, Chuntao Wang ${ }^{\dagger}$, Yuhang Ge and Tao Fu* \\ Department of Gastrointestinal Surgery II, Renmin Hospital of Wuhan University, Wuhan, China
}

Background: Recent papers have described circular RNAs (circRNAs) playing important roles in the development and progression of colorectal cancer (CRC). However, the expression profiles of circRNAs and their functions in CRC have rarely been studied. The objective was to identify circRNAs involved in the carcinogenesis and progression of $\mathrm{CRC}$ and to explore potential molecular mechanisms as a competitive endogenous RNA (ceRNA). Moreover, we aimed to establish an immune-related gene signature for predicting the overall survival (OS) of CRC.

Methods: The expression patterns of circRNA, miRNA, mRNA, and clinicopathological data were collected from the GEO and TCGA databases. A ceRNA network would be established, and the functional enrichment analyses were performed. The proteinprotein interaction network (PPI) was constructed, and hub genes were identified using a cytohub plugin. Subsequently, an immune-related signature was developed based on mRNAs in the ceRNA network. In addition, OS-nomogram was constructed by combining an immune-related signature and clinicopathological characterization to predict the OS.

Results: We established a circRNA-miRNA-mRNA ceRNA network. Kyoto Encyclopedia of Genes and Genomes (KEGG) pathway analysis revealed that the mRNAs were mainly enriched in neuroactive ligand-receptor interaction, Wnt signaling pathway, cell adhesion molecules (CAMs), and renin secretion. PPI network and module analysis identified 10 hub genes, and the circRNA-miRNA hub gene regulatory modules was established. After univariate and multivariate analysis, seven immune-related genes in the ceRNA network were used to construct the immune-related signature. Patients were divided into low-risk and high-risk groups, and there were significant differences in the OS. The ROC of the nomogram indicated the satisfactory accuracy and predictive power. Furthermore, we established a prognostic nomogram based on immune-related risk score and clinical characterization. The ROC and calibration curves revealed the accuracy of the nomogram. In addition, the high-risk score was positively correlated with six immune infiltrating cells $(P<0.05)$. 
Conclusion: We screened the key genes and established a circRNA-related ceRNA network involved in CRC, which will assist in understanding the molecular mechanisms underlying the carcinogenesis and progression. Moreover, our proposed immune-based signature may predict survival and reflect the immune status of CRC patients.

Keywords: colorectal cancer, circRNA, competitive endogenous RNA, immune-related genes, prognostic signature

\section{INTRODUCTION}

Colorectal cancer (CRC) is one of the most commonly diagnosed malignancies and one of the leading causes of death from cancer worldwide (Bray et al., 2018). The incidence of CRC is expected to increase to $160 \%$ by 2030 , with an estimated 2.1 million new cases and 1.1 million deaths per year (Arnold et al., 2017). Considerable progress has been made in in the comprehensive treatment modalities of CRC over the past few decades. However, due to its late detection and high recurrence rate, mortality remains high, and the OS remains poor (Roxburgh and McMillan, 2012; Ludmir et al., 2017). Thence, it is essential to explore more reliable markers to precisely stratify the risk of individual patients and to implement personalized treatment for CRC patients.

Circular RNAs (circRNAs) are a new identified class of noncoding RNAs with a covalent loop structure without the $5^{\prime}$-end cap and $3^{\prime}$-end ploy A tail. They can prevent their degradation by RNA exonuclease and confer a strong stability in specific cells (Wang et al., 2016). Numerous studies have indicated that abnormal expressions of circRNAs may be a potential mechanism for the development and progression of cancer (Braicu et al., 2019; Wu et al., 2019). Although the function mechanism of circRNAs remains unclear, accumulating evidence suggest circRNAs may completely bind with miRNAs to influence the interaction between miRNAs and mRNAs and contribute to the progression of tumors, which was called as competitive endogenous RNA (ceRNA) hypothesis (Salmena et al., 2011). For example, has_circ_0009361 was shown to be downregulated in CRC tissues. Mechanistically, has_circ_0009361 may sponge miR-582 to regulate adenomatous polyposis coli 2 (APC2) level to inhibite cell proliferation, EMT, migration, and invasion (Geng et al., 2019). Similarly, hsa_circ_0008035 can interact with YBX1 by the sponging of miR-375 in gastric cancer (Huang et al., 2019). However, the tumor microenvironment is very complex, and it is not ideal to research one or several relationships of ceRNA in isolation.

Currently, postoperative adjuvant therapy and prognosis were based on the comprehensive evaluation of the TNM staging system (Amin et al., 2017). However, it provides only limited information for the clinical prognostication, since even patients within the same stage display a strong heterogeneity for prognosis and treatment response. In addition, this system cannot predict the prognosis of individual patients. Therefore, it is necessary to continuously develop better methods to more accurately predict the survival of CRC. Recently, some nomograms have been widely used for predicting the prognosis of CRC (Fernández Montes et al., 2019; Qu et al., 2019). For example, Fernández Montes et al. (2019) developed a nomogram to predict 3-, 6-, and 12-month OS based on six variables, including the tumor site, ECOG PS, BRAF mutations, the number of metastatic sites, the response to first-line, and CEA. Despite significant progress in these studies, few have considered the use of genetic characteristics to construct a risk stratification system of CRC.

In the present study, we established the circRNA ceRNA network related to CRC according to bioinformatic prediction. From the ceRNA network, we identified immune-related genes and established an immune-related risk signature. In addition, the signature was assessed in the receiver operating characteristic (ROC) analysis. Finally, a nomogram containing an immune-related gene and clinicopathological features had also been established to evaluate the probability of survival rate in CRC patients.

\section{MATERIALS AND METHODS}

\section{Data Acquisition}

We downloaded the circRNA (GSE126095) gene expression profiles from the GEO database ${ }^{1}$. The dataset included 10 CRC tissues and 10 paired normal colorectal tissues. The data of miRNA-seq (539 CRC samples and nine normal samples), mRNA-seq (571 CRC samples and 44 sample tissues), and their clinical data of CRC patients was collected from the Cancer Genome Atlas ${ }^{2}$. Immunologically relevant list of genes was downloaded from the IMMPORT Database ${ }^{3}$. Given that all data were downloaded from the public databases, the current study was no required approval by ethics committee.

\section{Screening of DEGs}

The "limma" and "edgeR" package was used to identify differentially expressed genes (DEGs). Based on the cut-off value, adjusted $P$-values $<0.01$, and $\mid \log$ fold change (FC) $\mid$ $>3$ were used for selecting differentially expressed circRNAs (DEcircRNAs). The miRNAs and mRNAs that met the cut-off criteria of the adjusted $P$-values $<0.05$ and $|\log F C|>2$ were screened out as differentially expressed miRNAs (DEmiRNAs) and mRNAs (DEmRNAs).

\section{CeRNA Network Construction}

We used the Cancer-Specific CircRNA ${ }^{4}$ to screen the target miRNAs of the DEcircRNAs. These target miRNAs were further

\footnotetext{
${ }^{1}$ https://www.ncbi.nlm.nih.gov/geo/

${ }^{2}$ TCGA, https://cancergenome.nih.gov/

${ }^{3}$ https://www.immport.org/shared/genelists

${ }^{4}$ http://gb.whu.edu.cn/CSCD/
} 


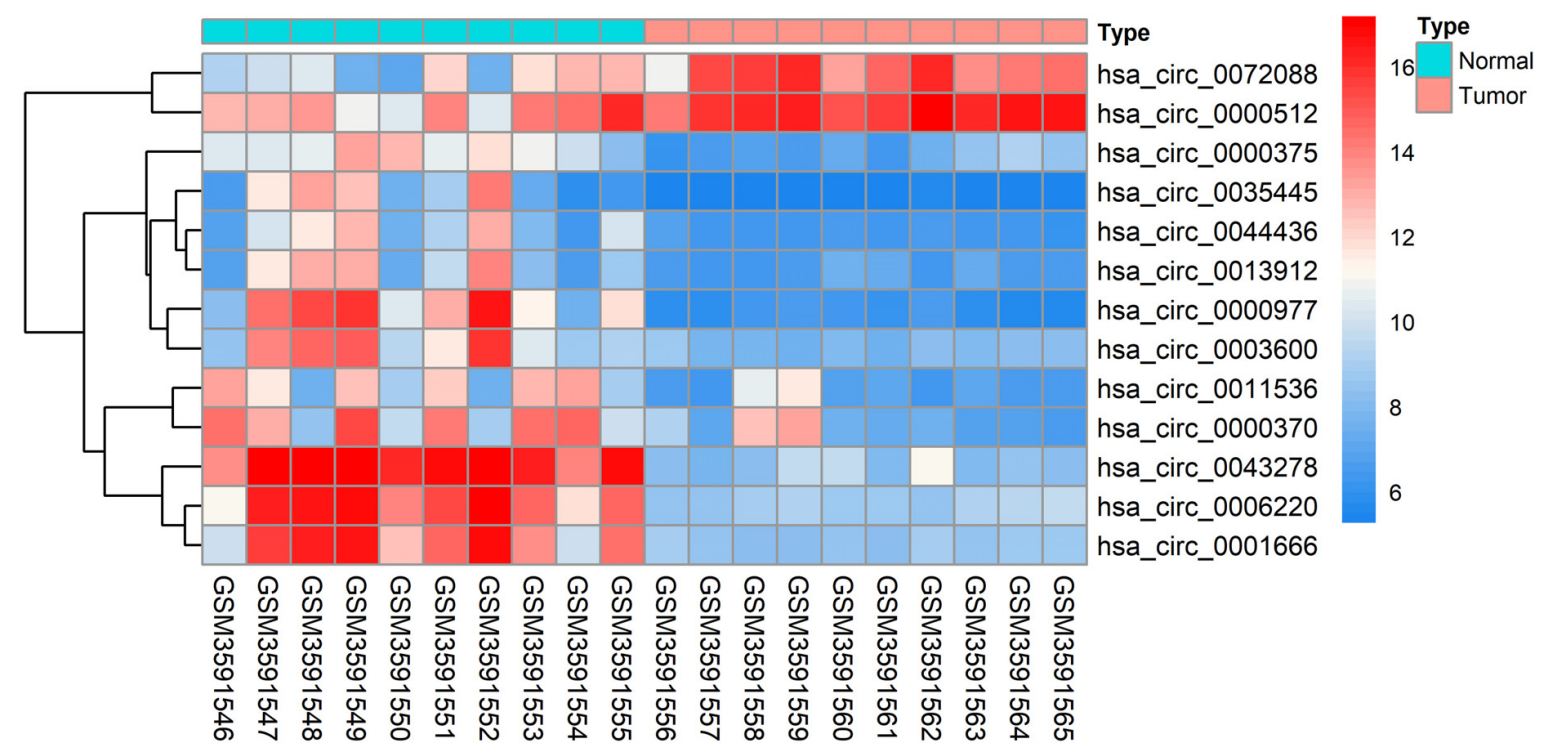

FIGURE 1 | Heatmap analysis of differentially expressed circRNAs.

screened by the DEmiRNAs obtained from TCGA database. Then, the target genes of these DEmiRNAs were predicted using the TargetScan, miRDB, and miRTarBase databases (Fromm et al., 2015; Wong and Wang, 2015). Only the target genes predicted by these three databases were retained, which were then overlapped with the DEmRNAs collected from the TCGA to obtain the CRC associated miRNA-mRNA interaction pairs. Finally, according to the predicted relationship of circRNA-miRNA and miRNA-mRNA, the ceRNA network was constructed using the Cytoscape software ${ }^{5}$.

\section{Functional Enrichment Analysis}

To screen the possible function of mRNAs in ceRNA network, we performed the Gene Ontology (GO) function and the Kyoto Encyclopedia of Genes and Genomes (KEGG) pathway enrichment analysis by "clusterProfiler" package in R. $P<0.05$ was considered statistically significant.

\section{PPI Network Construction and Identification of Hub Genes}

We used the Search Tool for the Retrieval of Interacting Genes (STRING) ${ }^{6}$ database (Wang et al., 2011) to establish the PPI network of DEmRNAs. In this study, we used interaction score greater than 0.9 as the cut-off criterion. Subsequently, the hub genes were identified by cytoHubba plug in the Cytoscape software (v 3.7.0).

\section{Signature Development and Validation}

To identify differentially expressed immune-related genes, we downloaded immune-related genes list, and intersected with the identified DEmRNAs in the ceRNA network.

\footnotetext{
${ }^{5}$ www.cytoscape.org/
}

${ }^{6} \mathrm{http}: / /$ string-db.org/
Only the shared genes were left. To discover potential immune-related genes that are significantly associated with the OS, we conducted the univariate Cox proportional hazards regression. Possible immune-related genes with a $P<0.05$ from univariate analysis were further included in a multivariate Cox regression analysis backward stepwise regression. An immunerelated signature was established by multivariate Cox regression analysis. The regression coefficients $(\beta)$ of each related gene are reserved for the development of immune-related signature. The risk score $=\sum \exp (m R N A) * \beta$. By the median risk score as the cut-off value, the patients were divided into high and low risk groups. The Kaplan-Meier survival analysis, time-dependent receiver operating characteristic (tdROC) curve analysis, and calibration curve analysis were used to evaluate predictive ability of the signature.

\section{Construction and Validation of Nomogram}

Based on the immune-related risk score and clinicopathological parameters, a prognostic nomogram was established by the "rms" software package in R. Discrimination between the outcomes of observations and predictions using the area under the curve (AUC) of ROC in the package of "survivalROC" in R. The value of the AUC was range 0.5 from 1.0 , with 0.5 indicating a random probability, and 1.0 indicating the perfect ability. A calibration map was generated by comparing the nomogram prediction probability and the observation for the 3-year and 5 -year OS rates. In addition, we compared predictive accuracy between the nomogram and a single independent factor by the ROC curve. The R software (version: 3.5.3) and SPSS 23.0 (Chicago, IL, United States) were applied to conduct all the analyses. Data was considered to be statistically significant when $P<0.05$. 


\section{Estimation of Tumor-Infiltrating Immune Cell Types}

Tumor Immune Estimation Resource TIMER, ${ }^{7}$ was used to analyze the correlation between tumor-infiltrating immune cells signatures and risk score. TIMER can systematically analyze immune infiltration of various types of cancer. The abundance of the six immune infiltrates was estimated by statistical methods and validated using pathological estimates. The six immune cells are composed of CD4+ T cells, CD8 $+\mathrm{T}$ cells, B cells, Macrophages, Neutrphils, and Dendritic cells.

\section{RESULTS}

\section{Identification of DEGs in CRC Patients}

The DEcircRNAs were identified by the "limma" package in $\mathrm{R}$ (adjusted $P<0.01$ and $|\log \mathrm{FC}|>3$ ). In total, 13 DEcircRNAs that included two upregulated circRNAs and 11 downregulated circRNAs were screened (Figure 1). Using the criteria of adjusted $P<0.02$ and $|\log \mathrm{FC}|>2,381$ DEmiRNAs (including 276 upregulated one and 105 downregulated ones), 3,933 DEmRNAs (including 2644 upregulated one and 1289 downregulated ones) were identified. A total of 2,498 immune-related genes were downloaded from the IMMPORT Database. We identified 91 differentially expressed immune-related genes of adjusted $p$-value $<0.05$ and $|\operatorname{logFC}|>2$, of which, 42 genes were downregulated, and 49 genes were up-regulated. The clinicopathologic characteristics of CRC patients are summarized in Table 1.

\section{Construction of ceRNA Network}

After searching the CSCD database, we identified 713 circRNAmiRNA pairs, including 13 DEcircRNAs and 549 miRNAs. After intersecting with the 381 DEmiRNAs, only 57 miRNAs remained. Subsequently, the target genes were predicted for these 57 miRNAs by TargetScan, miRDB, and miRTarBase databases, which were then overlapped with 3,933 DEmRNAs collected from the TCGA, resulting in 864 genes shared. On the basis of the combination of the above data, we established the ceRNA network (Figure 2).

\section{Functional Enrichment Analysis}

GO function annotation of the 864 DEmRNAs includes three parts: biological process (BP), molecular function (MF), and cell component (CC). As shown in Figure 3A, 589 GO terms (including $463 \mathrm{BP}, 64 \mathrm{CC}$, and $61 \mathrm{MF}$ ) were enriched. The muscle system process was significantly enriched for BP, while for CC, it was was extracellular matrix, and for MF it was the cation transmembrane transporter activity. Furthermore, we analyzed the KEGG pathways of these genes. The results showed 30 KEGG pathways were enriched (Figure 3B), mainly including neuroactive ligand-receptor interaction, Wnt signaling pathway, cell adhesion molecules (CAMs), and renin secretion. The top 20 KEGG pathways are listed in Figure 3B.

\footnotetext{
${ }^{7}$ https://cistrome.shinyapps.io/timer/
}

TABLE 1 | Clinicopathologic characteristics of CRC patients.

\begin{tabular}{|c|c|}
\hline Variables & Case, $n(\%)$ \\
\hline Age ( $M \pm S D$, years) & $65.81 \pm 12.45$ \\
\hline \multicolumn{2}{|l|}{ Gender } \\
\hline Female & $241(46.0)$ \\
\hline Male & $283(54.0)$ \\
\hline \multicolumn{2}{|l|}{ History of polyps } \\
\hline No & $309(59.0)$ \\
\hline Yes & $146(27.9)$ \\
\hline Unknown & $69(13.2)$ \\
\hline \multicolumn{2}{|c|}{ Presentation o metastases } \\
\hline No & $26(5.0)$ \\
\hline Yes & $455(86.8)$ \\
\hline Unknown & $43(8.2)$ \\
\hline \multicolumn{2}{|l|}{ Microsatellite instability } \\
\hline Yes & $8(1.5)$ \\
\hline No & $86(16.4)$ \\
\hline Unknown & $430(82.1)$ \\
\hline \multicolumn{2}{|l|}{ Tumor location } \\
\hline Colon & $451(86.1)$ \\
\hline Rectum & $73(13.9)$ \\
\hline \multicolumn{2}{|l|}{ Lymphatic invasion } \\
\hline No & $284(54.2)$ \\
\hline Yes & $189(36.1)$ \\
\hline Unknown & $51(9.7)$ \\
\hline \multicolumn{2}{|l|}{ Venous invasion } \\
\hline No & $345(65.8)$ \\
\hline Yes & $110(21.0)$ \\
\hline Unknown & 69 (13.2) \\
\hline \multicolumn{2}{|l|}{ Stage } \\
\hline । & $98(18.7)$ \\
\hline$\|$ & $199(38.0)$ \\
\hline III & $143(27.3)$ \\
\hline IV & $84(16.0)$ \\
\hline \multicolumn{2}{|c|}{ Response to first-line therapy } \\
\hline Complete response & $146(27.9)$ \\
\hline Other & $41(7.8)$ \\
\hline Unknown & $337(64.3)$ \\
\hline
\end{tabular}

Other: partial response, progressive disease, and stable disease.

\section{PPI Network Construction and Analysis of Modules}

To further explore the interaction among the 864 common DEGs, we established the PPI network (Figure 4A). The PPI network contained 862 nodes and 828 edges. Ten hub genes were identified based on their MCC score using the Cytoscape software. These screened hub genes were CASR, AGT, GNGT1, GNG7, HTR1D, PTGDR2, NPY2R, HCAR1, SSTR2, and P2RY12 (Figure 4B). Then, we established a circRNAmiRNA hub gene subnetwork. After excluding the RNAs with the opposite expression of the circRNAs and mRNAs, the subnetwork only contains including five circRNAs (hsa_circ_0035445, hsa_circ_0001666, and hsa_circ_0000375, hsa_circ_0003600, and hsa_circ_0000512), 7 miRNAs (hsa-miR-215-3p, hsa-miR-1855p, hsa-miR-3194-5p, hsa-miR-671-5p, hsa-miR-486-3p, hsamiR-3652, and hsa-miR-605-5p), and 7 mRNAs (P2RY12, SSTR2, CASR, NPY2R, GNG7, AGT, and PTGDR2) (Figure 4C). 


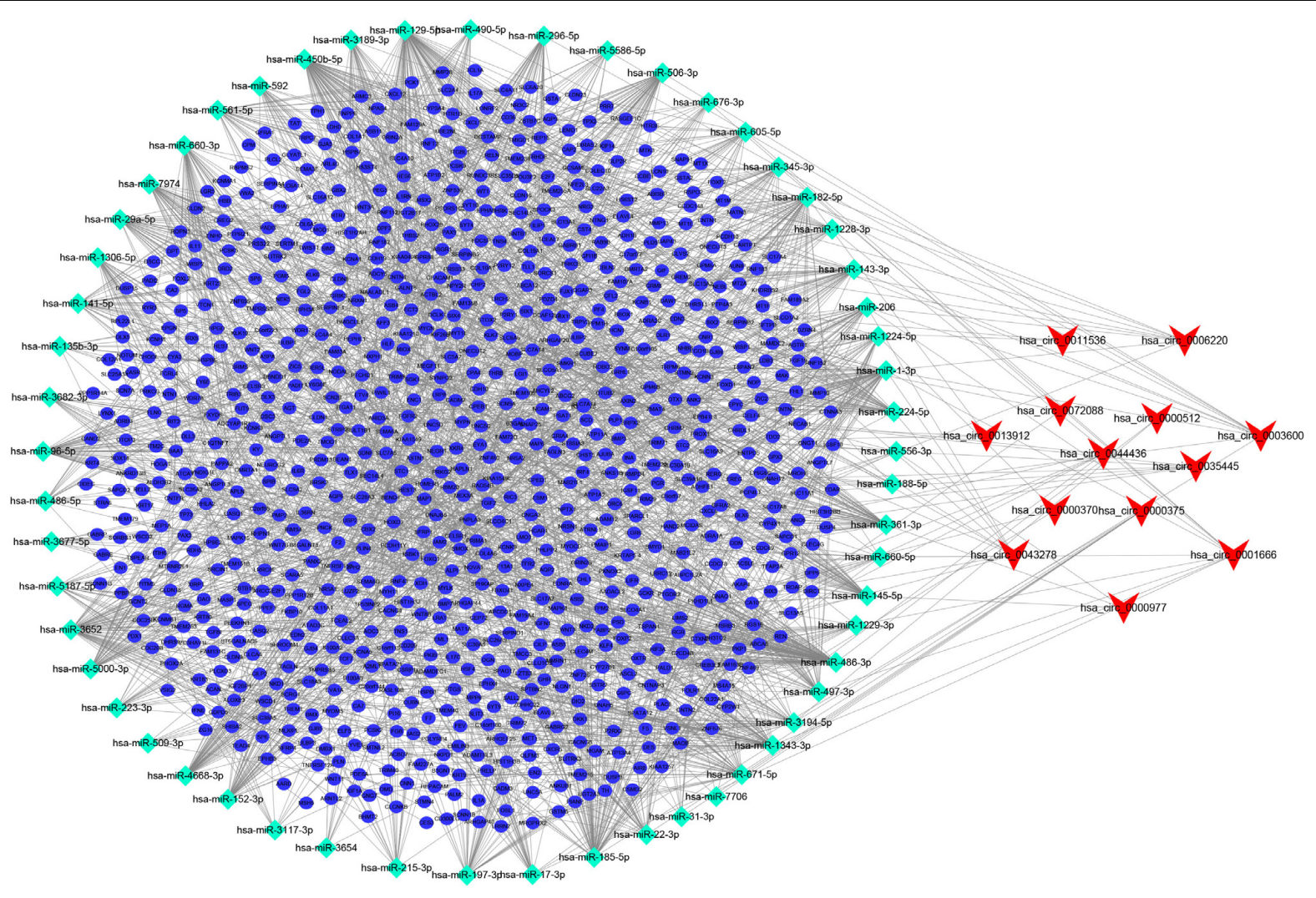

FIGURE 2 | circRNA-miRNA-mRNA ceRNA network in CRC patients. V, diamonds, and ellipses represent circRNAs, miRNAs and mRNAs, respectively.
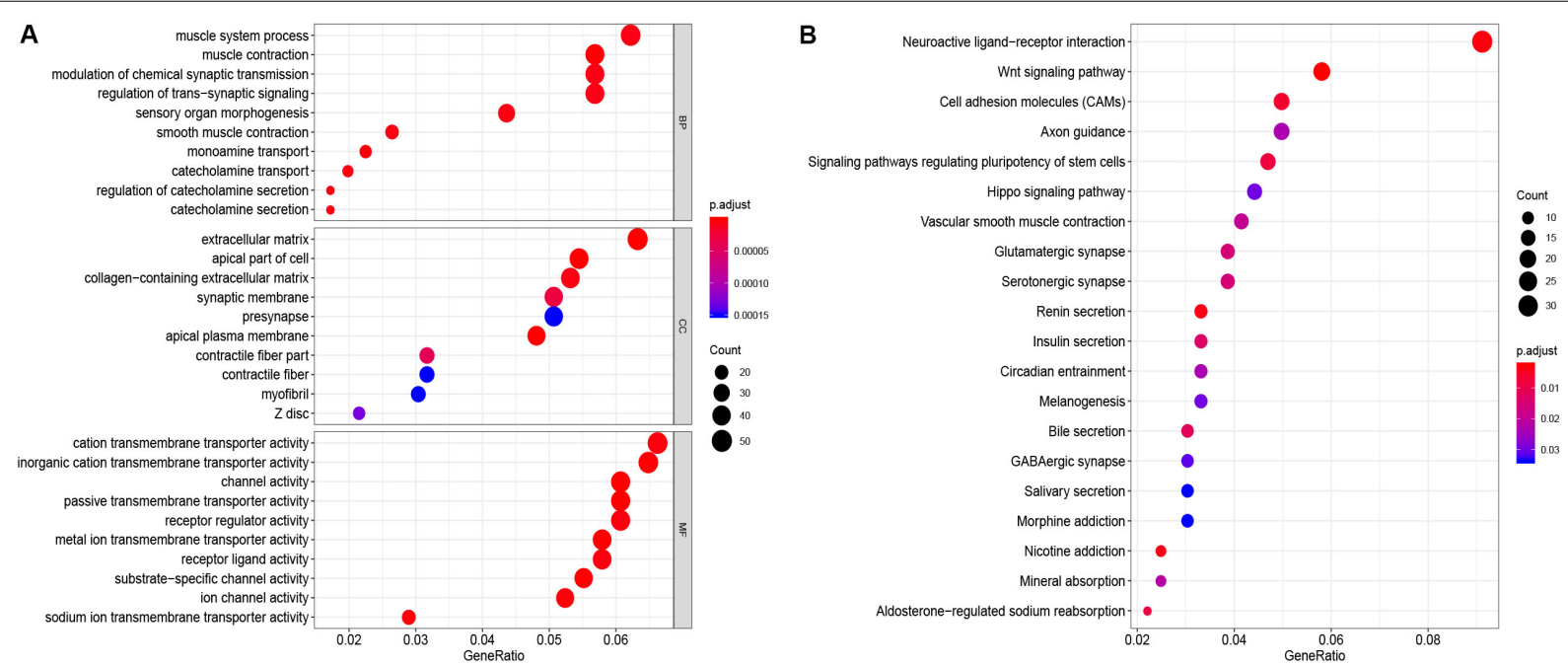

FIGURE 3 | Enrichment of top 10 GO terms (A) and KEGG pathways (B) of differentially expressed mRNAs. The node color changes gradually from blue to red in ascending order according to the adjust $p$-values. The size of the node represents the number of counts.

\section{Establishment of Immune-Related Signature}

Ninety-one differentially expressed immune-related genes were initially subjected to univariate analysis. The result showed that 10 immune-related genes were significantly correlated with the
OS $(P<0.05)$. Then, the 10 genes were further included in a multivariate analysis. Finally, seven immune-related genes (SEMA3E, CXCR5, JAG2, STC2, COLEC10, GLP2R, and NR3C2) were identified (Table 2). The different expressions of the 7 genes between CRC and normal colorectal tissues are listed 

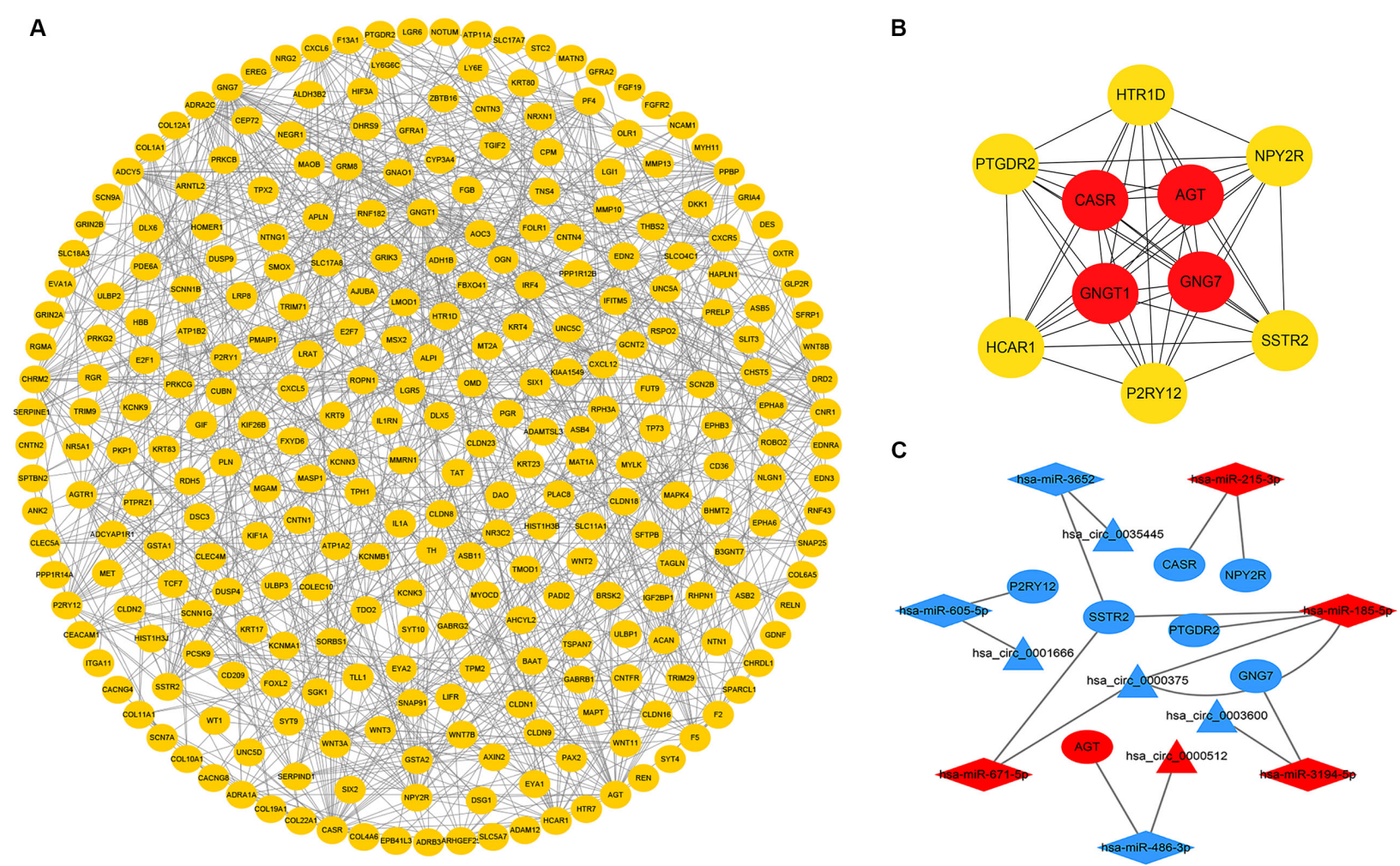

FIGURE 4 | Identification of hub genes from the PPI network and circRNA-miRNA hub gene ceRNA subnetwork. (A) a PPI network was constructed from STRING using the 864 common DEmRNAs; (B) PPI network of 10 hub genes extracted from A. The node color changes gradually from yellow to red in ascending order according to the log2(foldchange) of genes; (C) circRNA-miRNA hub gene ceRNA subnetwork. The nodes highlighted in red indicate upregulation, and the nodes highlighted in blue indicate downregulation. Triangles, diamonds, and ellipses represent circRNAs, miRNAs, and hub genes, respectively.

TABLE 2 | Seven prognostic mRNAs significantly associated with OS.

\begin{tabular}{|c|c|c|c|c|}
\hline Gene name & Coefficient & Down/up-regulated & Hazard ratio $(95 \% \mathrm{Cl})$ & $P$-value \\
\hline SEMA3E & 1.484163 & Up & $4.41(2.36-8.24)$ & $<0.001$ \\
\hline CXCR5 & 4.120982 & Up & $61.62(10.45-363.49)$ & $<0.001$ \\
\hline JAG2 & 0.033494 & Up & $1.03(1.01-1.06)$ & 0.005 \\
\hline STC2 & 0.028039 & Up & $1.03(0.99-1.07)$ & 0.143 \\
\hline COLEC10 & -2.26312 & Down & $0.10(0-2.51)$ & 0.163 \\
\hline GLP2R & -2.55344 & Down & $0.08(0.01-0.54)$ & 0.010 \\
\hline NR3C2 & -0.11606 & Down & $0.89(0.79-1.00)$ & 0.055 \\
\hline
\end{tabular}

in Figure 5. The risk score $=1.484163 *$ (expression value of SEMA3E) + 4.120982* (expression value of CXCR5) + $0.033494 *$ (expression value of JAG2) + 0.028039* (expression value of STC2) - 2.26312 * (expression value of COLEC10) 2.55344 * (expression value of GLP2R) - 0.11606 * (expression value of $N R 3 C 2$ ). The risk score for each patient was calculated and all patients were divided into high $(n=226)$ and low risk group $(n=227)$ based on the median risk score. The Kaplan-Meier log rank test illustrated that there were significant differences in OS $(P<0.001)$ (Figure 5). The AUCs for 3-year and 5-year OS were 0.701 and 0.728 , respectively (Figure 5). The risk score, overall survival time, Kaplan-Meier curve, and ROC curve of seven-gene signature were shown in Figure 5.

\section{Construction and Validation of Nomogram}

To determine whether the seven-gene signature could be an independent predictor of CRC patients, we performed the univariate and multivariate Cox regression analysis. Age, lymphatic invasion, venous invasion, response to first-line therapy, tumor stage, and risk score were significantly associated with the OS in the univariate analysis (Figure 6A). All the significant factors were selected into the multivariate Cox regression analysis. Only age, stage, response to first-line therapy, and risk score remained statistically significant $(P<0.05$; Figure 6B). A nomogram was then constructed to predict the 3-year and 5-year OS of CRC patients (Figure 6C). The AUC 


\section{A}

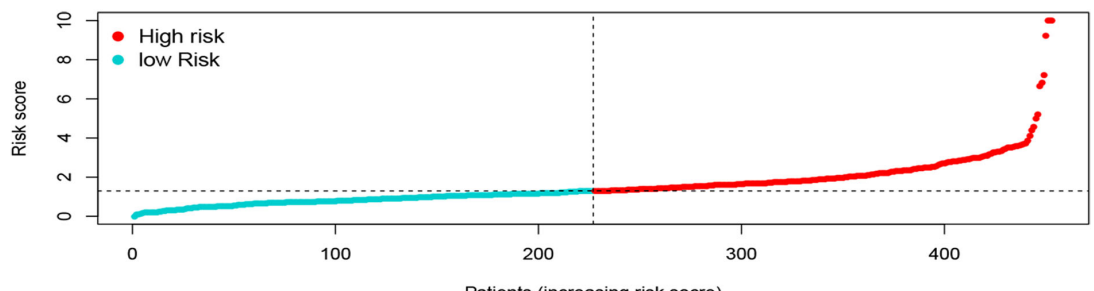

B

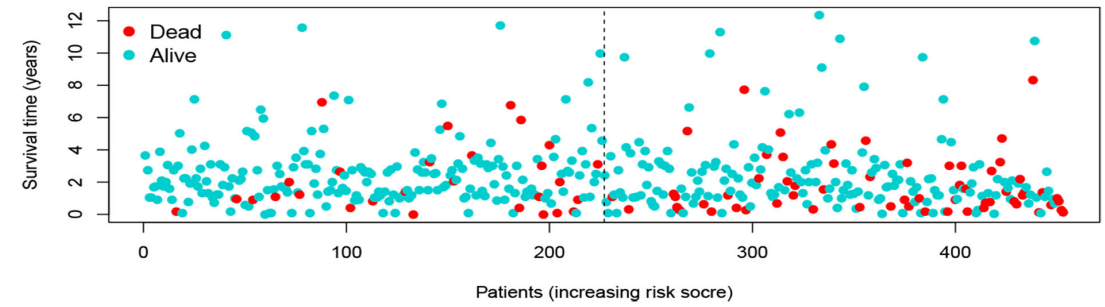

C

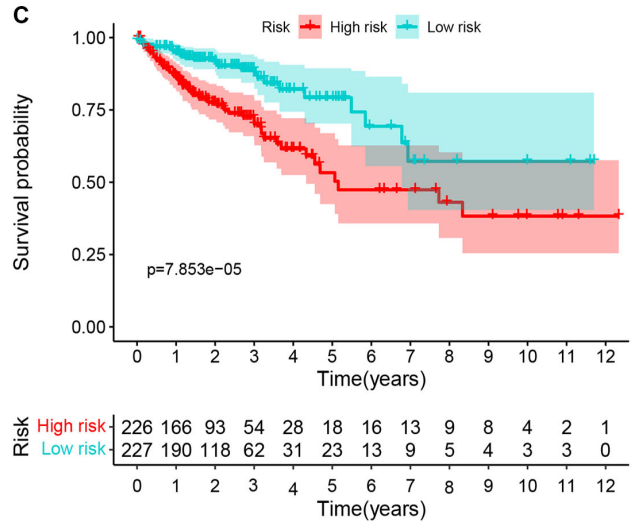

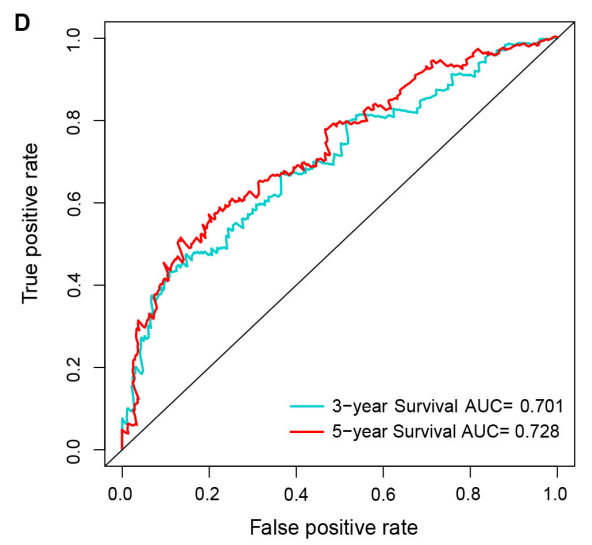

FIGURE 5 | The distribution of risk score (A), survival status (B), Kaplan-Meier curve (C), and ROC curve (D) of 7-immune-gene signature.

value for the 3-year (Figure 7A) and 5-year OS (Figure 7B) rates were 0.793 and 0.791 , respectively. Moreover, our result demonstrated that the predictive ability of the nomogram was significantly better than the TNM system (Figures 7A,B). The calibration curves for the probability of OS of 3 years or 5 years showed no deviations between the prediction by nomogram and actual reference line (Figures 7C,D).

\section{Immune Infiltration of Risk Score}

We evaluated the correlation between risk score and immune infiltration level by TIMER online resource. The result indicated that high-risk score was positively correlated with B cells, CD4+ $\mathrm{T}$ cells, CD8+ T cells, macrophages, neutrphils, and dendritic cells $(P<0.05)$, prompting a general increase in immune infiltration level (Figure 8).

\section{DISCUSSION}

In this study, we aimed to investigate possible crucial circRNAs impacting on development and progression in CRC patients. To comprehensively analyze the ceRNA network, we collected the RNA expression data from the GEO and TCGA databases, including circRNA, miRNA, and mRNA. We identified 13 DEcircRNAs that may act as a sponge of miRNAs to affect CRC progression via regulating the mRNAs. Based on the joint analysis of multiple databases, we established a CRCrelated ceRNA network. Then, we analyzed these 864 DEmRNA functions through the GO database and the KEGG pathway. In addition, PPI networks were also constructed to discover the hub genes. We established circRNA-miRNA hub gene network, including five circRNAs, seven miRNAs, and seven mRNAs. Moreover, we established seven immune-related signatures though immune genes in the ceRNA network. Patients with high-risk had a significantly worse OS than low-risk patients. The Kaplan-Meier curve illustrated that there were significant differences in the OS between high-risk and low-risk groups. The ROC curve demonstrated good performance. Furthermore, we established a prognostic nomogram that combines immunerelated risk scores and clinicopathological features. The accuracy of the nomogram for predicting 3-year and 5-year OS was confirmed by the discrimination and calibration plots. Finally, we approved the correlation between risk score and six immune infiltrates. 


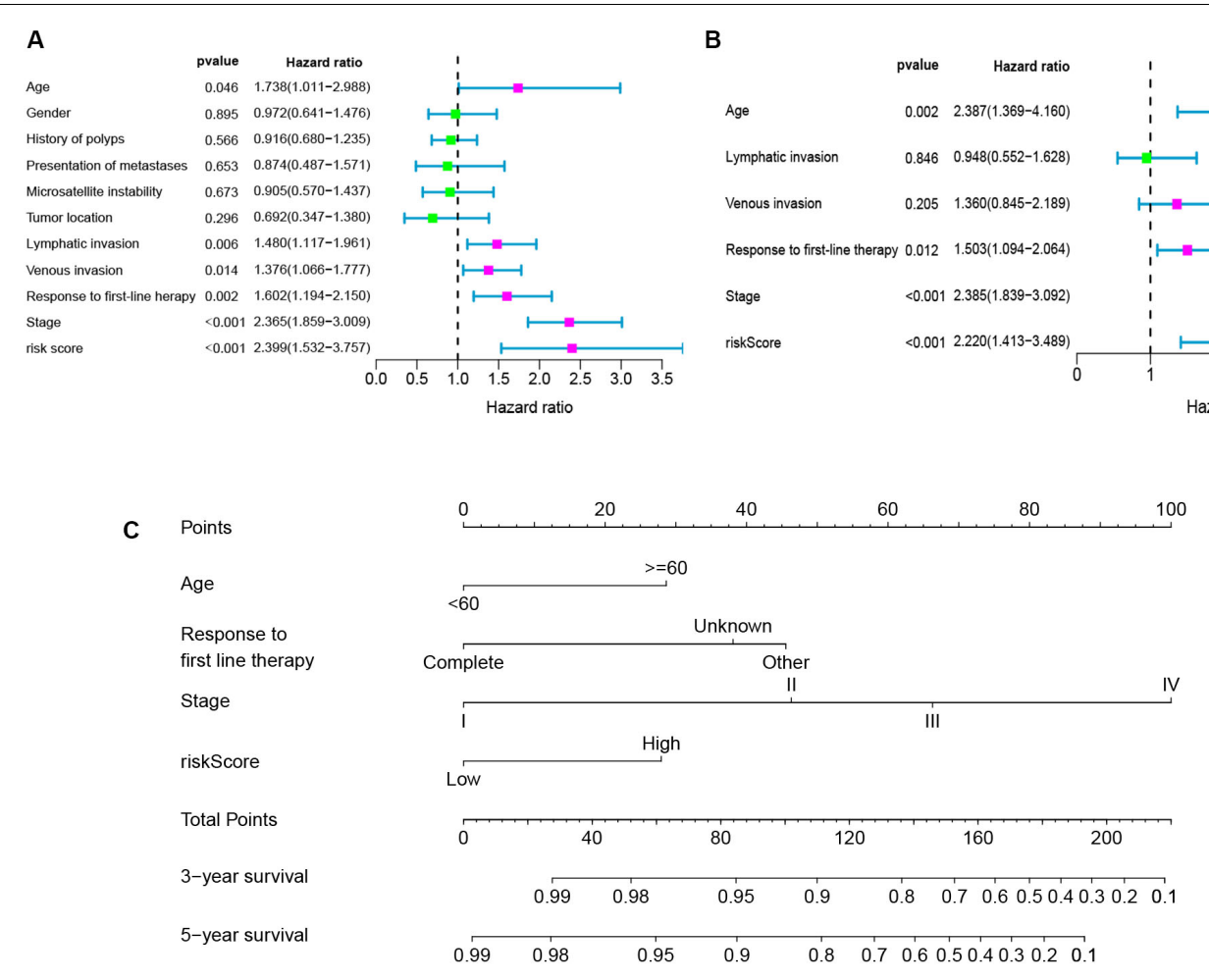

FIGURE 6 | Univariate analyses (A), multivariate analyses (B), and nomogram (C) for predicting 3- and 5-year OS of CRC patients.

With the development of high-throughput sequencing technology, more and more circRNAs were found to be abnormally expressed in tumor tissues and to have a critical impact on tumor progression (Wu et al., 2019). Although the functional mechanism of circRNAs remains unclear, accumulating evidence indicates that circRNAs can act as a miRNA sponge to influence the interactions between miRNAs and mRNAs and contribute to the progression of CRC. For example, hsa_circRNA_102958 was found to be upregulated in CRC tissues. Silence of hsa_circRNA_102958 can inhibit CRC growth, migration, and invasion. Further studies revealed that hsa_circRNA_102958 could exert its oncogenic functions by sponging miR-585 and lead to the release of its repression on CDC25B (Ruan et al., 2019). Similarly, Yang et al. indicated that circ-ITGA7 was downregulated in CRC tissues. Mechanistically, circ-ITGA7 might sponge miR-3187-3p to increase ASXL1 level to suppress cell proliferation, migration, and invasion ( $\mathrm{Li} \mathrm{R}$. et al., 2019). In our study, there were five crucial circRNAs (hsa_circ_0001666, hsa_circ_0035445, and hsa_circ_0000375, hsa_circ_0003600, and hsa_circ_0000512) included in the subnetwork. Afzali and Salimi (2019) constructed a circRNAmiRNA network by microarray analysis and found that hsa_circ_0001666 was down-regulated in breast cancer tissues. However, they did not conduct functional studies. Similarly, hsa_circ_0035445 was highly expressed in gastric cancer tissue by microarray analysis (Shao et al., 2017). However, none of these five circRNAs was reported in the CRC. Therefore, their role in CRC needs to be further verified in future studies.
MiRNAs are types of endogenous noncoding RNA comprising about approximately 19-25 nt long, with widespread participation physiological activity. Studies have found that miRNAs play crucial roles in various pathological processes, and miRNAs have been identified as oncogenes or tumor suppressor genes in regulating cancer progression (Manikandan et al., 2008). MiR-215-3p was found to be downregulated in the 5-FU resistant cell, and high miR-215-3p expression sensitized CRC to 5-fluorouracil-induced apoptosis by regulating the expression of CXCR1 (Li et al., 2018). Consistent with previous studies, we found a higher expression of miR-215-3p in CRC patients. Zhu et al. (2018) found that lncRNA FOXD2-AS1 could promote proliferation and migration of CRC though interacting with miRNA-185-5p. Della Vittoria Scarpati et al. (2012) confirmed by qRT-PCR that miR-671-5p is significantly upregulated in rectal cancer patients. However, miR-3194$5 p$, miR-486-3p, and miR-3652 have not been reported in patients with CRC.

In our circRNA-miRNA hub gene subnetwork, seven hub genes with the highest connective degree were selected using the MCC method. The hub genes are P2RY12, SSTR2, CASR, NPY2R, GNG7, AGT, and PTGDR2. Some of these genes have been reported to be closely related to CRC, such as SSTR2, CASR, and AGT (Li et al., 2017; Momen-Heravi et al., 2017; Patnaik and Anupriya, 2019). Several studies have found that SSTR2 is methylated in CRC tissues, suggesting that SSTR2 may serve as a potential epigenetic marker for early detection of CRC (Li et al., 2017). Momen-Heravi et al. (2017) found that overexpression of 

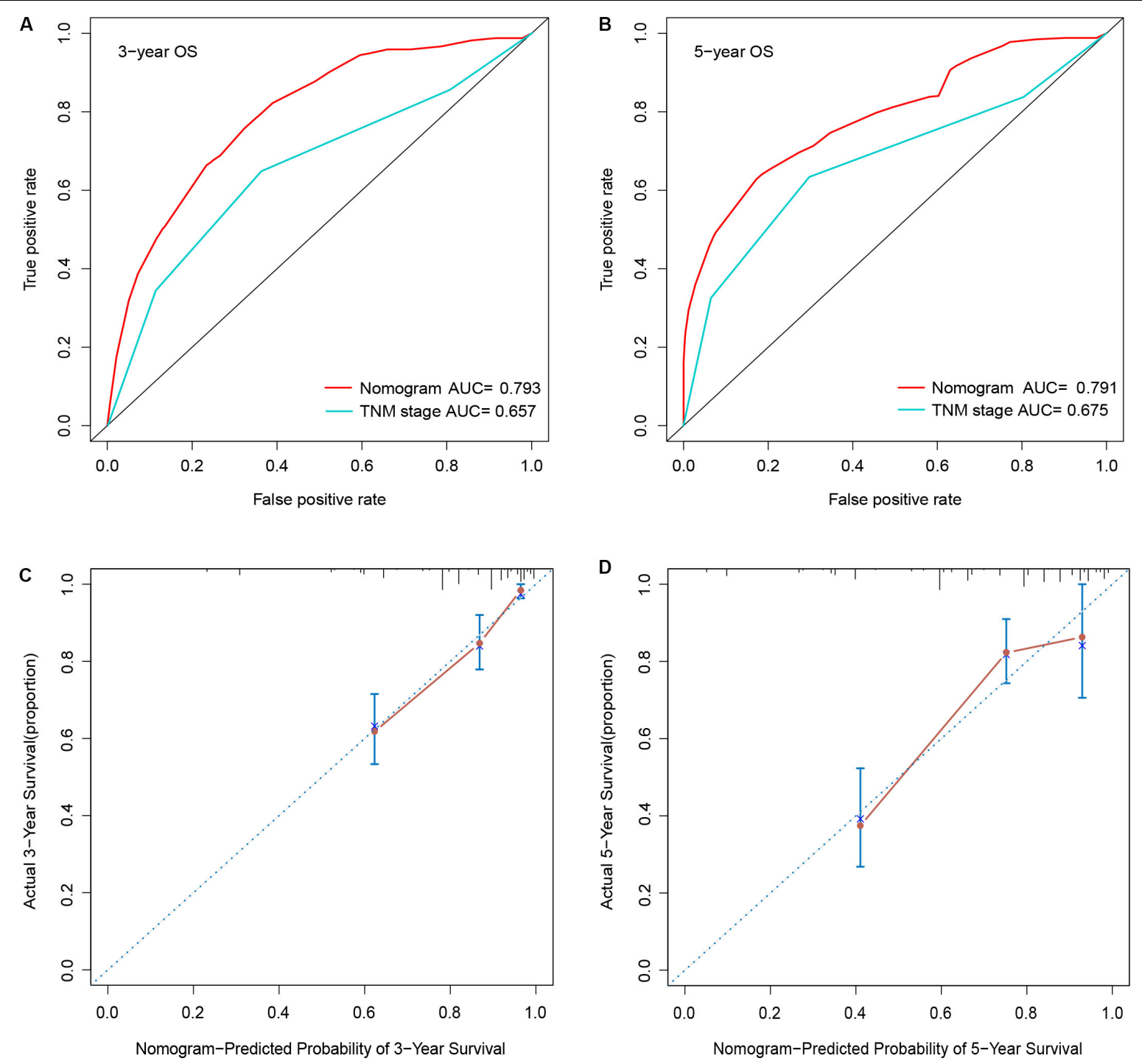

FIGURE 7 | Comparison of the AUCs of the nomogram and AJCC TNM staging system, and calibration curves of the nomogram of patients with CRC. (A) 3-year AUC; (B) 5-year AUC; (C) 3-year calibration curves; (D) 5-year calibration curves.

CASR was correlated with a lower risk of CRC-specific mortality. Martin et al. (2014) indicated that $A G T$ epithelial expression was associated with a longer progression free survival, suggesting that $A G T$ may be a prognostic marker in metastatic CRC patients treated with chemotherapy and bevacizumab.

KEGG enrichment analyses using DEmRNAs provided an intuitive overview in elucidating the mechanism of CRC. Results suggested that the DEmRNAs were mostly enriched in neuroactive ligand-receptor interaction, the Wnt signaling pathway, cell adhesion molecules (CAMs), and renin secretion. These pathways play vital roles in the development of CRC. For example, Wnt signaling is constitutively overexpressed and is thought to be a crucial step in the development of CRC. Accumulated $\beta$-catenin enters the nucleus of colon cancer cells and interacts with $\mathrm{T}$ cell factor family transcription factors, leading to activation of downstream target genes of the Wnt pathway and promoting tumorigenesis (Barker and Clevers, 2006). Dong et al. revealed that the secreted V-set and transmembrane domain containing 2A (VSTM2A) significantly inhibits the Wnt signaling pathway in colon cancer cells. VSTM2A can inhibit the phosphorylation of Wnt signaling coreceptor LDL receptor-associated protein 6 (LRP6) and induce LRP6 endocytosis and lysosomal-mediated degradation, which together lead to the inactivation of Wnt signaling. Maintenance of cell adhesion, promotion, or destruction are particularly important process in colorectal tumorigenesis. Paschos et al. (2009) demonstrated that CAMs play key roles in the progression of CRC and its metastasis to the liver. CAMs are not only related to the detachment of malignant cells from the primary tumor, but also to the attachment of tumor cells to distant tissues. All these findings indicated the development of CRC might be related to these pathways. 
A

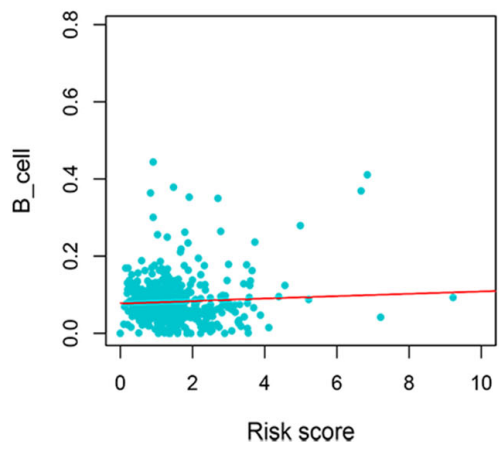

D

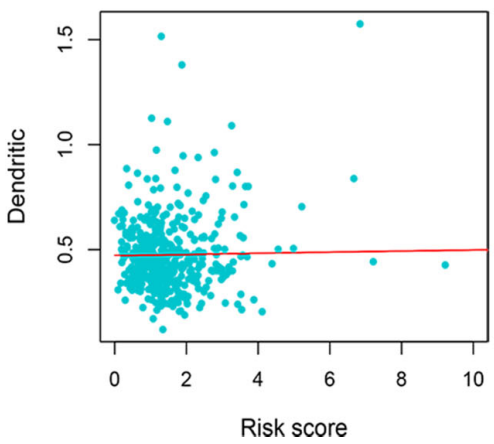

B

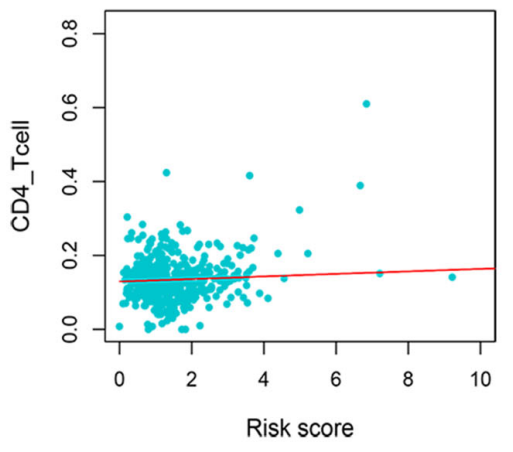

E

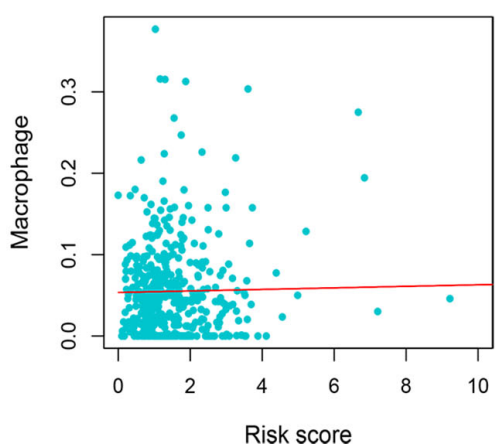

C

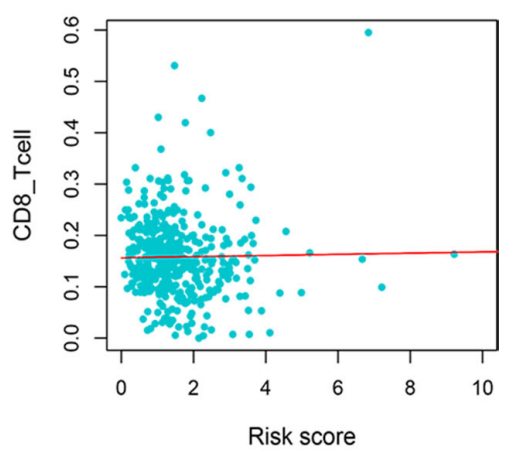

$\mathbf{F}$

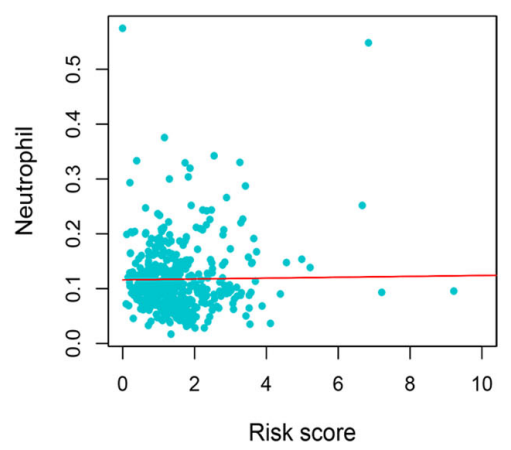

FIGURE 8 | Immune infiltration of 7-immune-gene signature. (A) B cells; (B) CD4+ T cells; (C) CD8+ T cells; (D) dendritic cells; (E) macrophages; (F) neutrophils.

Our proposed immune-related signature was significantly associated with OS of CRC patients. The signature constated of seven immune-related genes. Of these seven genes, four are potential risky genes and three are potential protective genes. All four risky genes have been previously reported to have a clear correlation with CRC. Semaphorin 3E (SEMA3E) was originally identified as a neurochemical chemotactic agent involved in regulating cell migration, proliferation and angiogenesis (Yong et al., 2016). Studies have indicated that SEMA3E plays a role in the regulation of various stages of cancer development. Kang et al. (2016) showed that miR-4282 inhibits CRC cell proliferation by targeting Sema3E by inhibiting its translation or enhancing Sema3E mRNA degradation. Yan et al. (2015) analyzed the expression of CXCR5 in 236 cases of CRC and adjacent normal tissue, and found that high CXCR5 expressions could promote the pathogenesis, metastasis and recurrence, suggesting that it can be used as a valuable predictor for metastasis and recurrence of CRC. Similarly, JAG2 and STC2 act as oncogenes to promote CRC development and progression through different signaling pathways, such as noncanonical Notch, non-EMT-dependent, and Wnt/ $\beta$-catenin pathway (He et al., 2019; Li Q. et al., 2019). However, the other three protective genes are rarely reported in CRC. Since a single gene may be predictive of instability in predicting the OS, the signature that integrate the efficacy of seven immune-related genes will show robust predictive power.

In order to establish a relatively accurate prognostic model in CRC patients, a novel prognostic nomogram including immune-related risk score and clinicopathological feature had been proposed in this study, which had displayed favorable predictive capability. In addition, significant correlation between the immune risk score and six immune infiltrating cells were observed. We speculate that there may be a link between the signature and response to immunotherapy. NF- $\mathrm{kB}$ is a key component of the immune response and cancer (Karin and Greten, 2005). Studies have shown that inhibition of NF- КBcRel attenuates regulatory $\mathrm{T}$ cell-mediated immunosuppression and enhances the therapeutic efficacy of anti-PD-1 (GrinbergBleyer et al., 2017). Of the seven immune-related genes, three genes are associated with NF- $\kappa \mathrm{B}$ activity (Deng et al., 2000; Kermarrec et al., 2019; Zhang et al., 2019). Therefore, it is further shown that the signature was associated with the response to immunotherapy. Nevertheless, the present study included some limitations. First, the circRNA-miRNAmRNA network was established by bioinformatics analysis. Experimental studies should be conducted to confirm our results. In addition, the performance of the seven-immunegene signature and nomogram need to be validated by additional data.

\section{CONCLUSION}

Our study identified five crucial circRNAs and established a circRNA-related ceRNA network involved in CRC, which will 
assist in understanding the molecular mechanisms underlying the carcinogenesis and progression. Moreover, a seven-immunebased signature were established. The signature might improve the prediction of survival and reflect the immune status and help surgeons to better improve risk stratification and choose the optimal treatments for patients with CRC.

\section{DATA AVAILABILITY STATEMENT}

Publicly available datasets were analyzed in this study. These data can be found in the Cancer Genome Atlas (https:// portal.gdc.cancer.gov/) and the NCBI Gene Expression Omnibus (GSE126095).

\section{ETHICS STATEMENT}

Ethical review and approval was not required for the study on human participants in accordance with the local legislation

\section{REFERENCES}

Afzali, F., and Salimi, M. (2019). Unearthing regulatory axes of breast cancer circRNAs networks to find novel targets and fathom pivotal mechanisms. Interdiscip. Sci. 11, 711-722. doi: 10.1007/s12539-019-00339-6

Amin, M. B., Greene, F. L., Edge, S. B., Compton, C. C., Gershenwald, J. E., Brookland, R. K., et al. (2017). The eighth edition AJCC cancer staging manual: continuing to build a bridge from a population-based to a more "personalized" approach to cancer staging. CA Cancer J. Clin. 67, 93-99. doi: 10.3322/caac. 21388

Arnold, M., Sierra, M. S., Laversanne, M., Soerjomataram, I., Jemal, A., and Bray, F. (2017). Global patterns and trends in colorectal cancer incidence and mortality. Gut 66, 683-691. doi: 10.1136/gutjnl-2015-310912

Barker, N., and Clevers, H. (2006). Mining the Wnt pathway for cancer therapeutics. Nat. Rev. Drug Discov. 5, 997-1014. doi: 10.1038/nrd2154

Braicu, C., Zimta, A. A., Harangus, A., Iurca, I., Irimie, A., Coza, O., et al. (2019). The function of non-coding RNAs in lung cancer tumorigenesis. Cancers (Basel) 11:605. doi: 10.3390/cancers11050605

Bray, F., Ferlay, J., Soerjomataram, I., Siegel, R. L., Torre, L. A., and Jemal, A. (2018). Global cancer statistics 2018: GLOBOCAN estimates of incidence and mortality worldwide for 36 cancers in 185 countries. CA Cancer J. Clin. 68, 394-424. doi: 10.3322/caac.21492

Della Vittoria Scarpati, G., Falcetta, F., Carlomagno, C., Ubezio, P., Marchini, S., De Stefano, A., et al. (2012). A specific miRNA signature correlates with complete pathological response to neoadjuvant chemoradiotherapy in locally advanced rectal cancer. Int. J. Radiat. Oncol. Biol. Phys. 83, 1113-1119. doi: 10.1016/j.ijrobp.2011.09.030

Deng, Y., Madan, A., Banta, A. B., Friedman, C., Trask, B. J., Hood, L., et al. (2000). Characterization, chromosomal localization, and the complete $30-\mathrm{kb}$ DNA sequence of the human Jagged2 (JAG2) gene. Genomics 63, 133-138. doi: 10.1006/geno.1999.6045

Fernández Montes, A., López López, C., Argilés Martínez, G., Páez López, D., López Muñoz, A. M., García Paredes, B., et al. (2019). Prognostic nomogram and patterns of use of FOLFIRI-aflibercept in advanced colorectal cancer: a realworld data analysis. Oncologist 24, e687-e695. doi: 10.1634/theoncologist.20180824

Fromm, B., Billipp, T., Peck, L. E., Johansen, M., Tarver, J. E., King, B. L., et al. (2015). A uniform system for the annotation of vertebrate microRNA genes and the evolution of the human microRNAome. Annu. Rev. Genet. 49, 213-242. doi: 10.1146/annurev-genet-120213-092023

Geng, Y., Zheng, X., Hu, W., Wang, Q., Xu, Y., He, W., et al. (2019). Hsa_circ_0009361 acts as the sponge of miR-582 to suppress colorectal cancer and institutional requirements. Written informed consent for participation was not required for this study in accordance with the national legislation and the institutional requirements.

\section{AUTHOR CONTRIBUTIONS}

WS and TF conceived and designed the original study. WS, JR, and CW collected and analyzed the data. WS, JR, CW, and YG contributed to the interpretation of the data. WS, JR, CW, and YG drafted the manuscript. WS and TF revised the manuscript. All authors saw and approved the final version of the manuscript.

\section{FUNDING}

This work was supported by the Suzhou Youth Science and Technology Foundation (KJXW2018032).

progression by regulating APC2 expression. Clin. Sci. 133, 1197-1213. doi: $10.1042 /$ cs20190286

Grinberg-Bleyer, Y., Oh, H., Desrichard, A., Bhatt, D. M., Caron, R., Chan, T. A., et al. (2017). NF-кB c-Rel Is crucial for the regulatory T cell immune checkpoint in cancer. Cell 170, 1096-1108.e13. doi: 10.1016/j.cell.2017.08.004

He, W., Tang, J., Li, W., Li, Y., Mei, Y., He, L., et al. (2019). Mutual regulation of JAG2 and PRAF2 promotes migration and invasion of colorectal cancer cells uncoupled from epithelial-mesenchymal transition. Cancer Cell Int. 19:160. doi: 10.1186/s12935-019-0871-5

Huang, S., Zhang, X., Guan, B., Sun, P., Hong, C. T., Peng, J., et al. (2019). A novel circular RNA hsa_circ_0008035 contributes to gastric cancer tumorigenesis through targeting the miR-375/YBX1 axis. Am. J. Transl. Res. 11, 2455-2462.

Kang, X., Wang, M., Wang, H., Shen, X., and Guan, W. (2016). MiR-4282 suppresses proliferation and mobility of human colorectal carcinoma cells by targeting semaphorin 3E. Panminerva Med. 58, 197-205.

Karin, M., and Greten, F. R. (2005). NF-kappaB: linking inflammation and immunity to cancer development and progression. Nat. Rev. Immunol. 5, 749-759. doi: 10.1038/nri1703

Kermarrec, L., Eissa, N., Wang, H., Kapoor, K., Diarra, A., Gounni, A. S., et al. (2019). Semaphorin-3E attenuates intestinal inflammation through the regulation of the communication between splenic CD11C and CD4 CD25 T-cells. Br. J. Pharmacol. 176, 1235-1250. doi: 10.1111/bph.14614

Li, J., Chen, C., Bi, X., Zhou, C., Huang, T., Ni, C., et al. (2017). DNA methylation of CMTM3, SSTR2, and MDFI genes in colorectal cancer. Gene 630, 1-7. doi: 10.1016/j.gene.2017.07.082

Li, Q., Zhou, X., Fang, Z., and Pan, Z. (2019). Effect of STC2 gene silencing on colorectal cancer cells. Mol. Med. Rep. 20, 977-984. doi: 10.3892/mmr.2019. 10332

Li, R., Wu, B., Xia, J., Ye, L., and Yang, X. (2019). Circular RNA hsa_circRNA_102958 promotes tumorigenesis of colorectal cancer via miR585/CDC25B axis. Cancer Manag. Res. 11, 6887-6893. doi: 10.2147/cmar. S212180

Li, X. W., Qiu, S. J., and Zhang, X. (2018). Overexpression of miR-215-3p sensitizes colorectal cancer to 5-fluorouracil induced apoptosis through regulating CXCR1. Eur. Rev. Med. Pharm. Sci. 22, 7240-7250. doi: 10.26355/eurrev_ 201811_16258

Ludmir, E. B., Palta, M., Willett, C. G., and Czito, B. G. (2017). Total neoadjuvant therapy for rectal cancer: an emerging option. Cancer 123, 1497-1506. doi: 10.1002/cncr.30600

Manikandan, J., Aarthi, J. J., Kumar, S. D., and Pushparaj, P. N. (2008). Oncomirs: the potential role of non-coding microRNAs in understanding cancer. Bioinformation 2, 330-334. doi: 10.6026/97320630002330 
Martin, P., Noonan, S., Mullen, M. P., Scaife, C., Tosetto, M., Nolan, B., et al. (2014). Predicting response to vascular endothelial growth factor inhibitor and chemotherapy in metastatic colorectal cancer. BMC Cancer 14:887. doi: 10.1186/1471-2407-14-887

Momen-Heravi, F., Masugi, Y., Qian, Z. R., Nishihara, R., Liu, L., Smith-Warner, S. A., et al. (2017). Tumor expression of calcium sensing receptor and colorectal cancer survival: results from the nurses' health study and health professionals follow-up study. Int. J. Cancer 141, 2471-2479. doi: 10.1002/ijc.31021

Paschos, K. A., Canovas, D., and Bird, N. C. (2009). The role of cell adhesion molecules in the progression of colorectal cancer and the development of liver metastasis. Cell. Signal. 21, 665-674. doi: 10.1016/j.cellsig.2009.01.006

Patnaik, S., and Anupriya (2019). Drugs targeting epigenetic modifications and plausible therapeutic strategies against colorectal cancer. Front. Pharmacol. 10:588. doi: 10.3389/fphar.2019.00588

Qu, A., Wang, W., Yang, Y., Zhang, X., Dong, Y., Zheng, G., et al. (2019). A serum piRNA signature as promising non-invasive diagnostic and prognostic biomarkers for colorectal cancer. Cancer Manag. Res. 11, 3703-3720. doi: 10. 2147/cmar.S193266

Roxburgh, C. S., and McMillan, D. C. (2012). The role of the in situ local inflammatory response in predicting recurrence and survival in patients with primary operable colorectal cancer. Cancer Treat. Rev. 38, 451-466. doi: 10. 1016/j.ctrv.2011.09.001

Ruan, H., Deng, X., Dong, L., Yang, D., Xu, Y., Peng, H., et al. (2019). Circular RNA circ_0002138 is down-regulated and suppresses cell proliferation in colorectal cancer. Biomed. Pharm. 111, 1022-1028. doi: 10.1016/j.biopha.2018.12.150

Salmena, L., Poliseno, L., Tay, Y., Kats, L., and Pandolfi, P. P. (2011). A ceRNA hypothesis: the rosetta stone of a hidden RNA language? Cell 146, 353-358. doi: 10.1016/j.cell.2011.07.014

Shao, Y., Li, J., Lu, R., Li, T., Yang, Y., Xiao, B., et al. (2017). Global circular RNA expression profile of human gastric cancer and its clinical significance. Cancer Med. 6, 1173-1180. doi: 10.1002/cam4.1055

Wang, F., Nazarali, A. J., and Ji, S. (2016). Circular RNAs as potential biomarkers for cancer diagnosis and therapy. Am. J. Cancer Res. 6, 1167-1176.

Wang, X., Yue, J., Ren, X., Wang, Y., Tan, M., Li, B., et al. (2011). Modularity analysis based on predicted protein-protein interactions provides new insights into pathogenicity and cellular process of Escherichia coli O157:H7. Theor. Biol. Med. Model. 8:47. doi: 10.1186/1742-4682-8-47

Wong, N., and Wang, X. (2015). miRDB: an online resource for microRNA target prediction and functional annotations. Nucleic Acids Res. 43, D146-D152. doi: 10.1093/nar/gku1104

Wu, J., Qi, X., Liu, L., Hu, X., Liu, J., Yang, J., et al. (2019). Emerging epigenetic regulation of circular RNAs in human cancer. Mol. Ther. Nucleic Acids 16, 589-596. doi: 10.1016/j.omtn.2019.04.011

Yan, Q., Yuan, Y., Yankui, L., Jingjie, F., Linfang, J., Yong, P., et al. (2015). The expression and significance of CXCR5 and MMP-13 in colorectal cancer. Cell Biochem. Biophys. 73, 253-259. doi: 10.1007/s12013-0150624-6

Yong, L. K., Lai, S., Liang, Z., Poteet, E., Chen, F., van Buren, G., et al. (2016). Overexpression of Semaphorin-3E enhances pancreatic cancer cell growth and associates with poor patient survival. Oncotarget 7, 87431-87448. doi: 10.18632/ oncotarget.13704

Zhang, G., Miao, F., Xu, J., and Wang, R. (2019). Mesenchymal stem cells from bone marrow regulate invasion and drug resistance of multiple myeloma cells by secreting chemokine CXCL13. Bosn. J. Basic Med. Sci. 20, 209-217. doi: 10.17305/bjbms.2019.4344

Zhu, Y., Qiao, L., Zhou, Y., Ma, N., Wang, C., and Zhou, J. (2018). Long non-coding RNA FOXD2-AS1 contributes to colorectal cancer proliferation through its interaction with microRNA-185-5p. Cancer Sci. 109, 2235-2242. doi: 10.1111/ cas. 13632

Conflict of Interest: The authors declare that the research was conducted in the absence of any commercial or financial relationships that could be construed as a potential conflict of interest.

Copyright (c) 2020 Song, Ren, Wang, Ge and Fu. This is an open-access article distributed under the terms of the Creative Commons Attribution License (CC BY). The use, distribution or reproduction in other forums is permitted, provided the original author(s) and the copyright owner(s) are credited and that the original publication in this journal is cited, in accordance with accepted academic practice. No use, distribution or reproduction is permitted which does not comply with these terms. 